\title{
Structure and activity relationships of the anti-Mycobacterium antibiotics resorcinomycin and pheganomycin
}

\author{
Yasushi Ogasawara, Koichi Ooya, Michiko Fujimori, Motoyoshi Noike and Tohru Dairi \\ The Journal of Antibiotics (2016) 69, 119-120; doi:10.1038/ja.2015.88; published online 26 August 2015
}

Tuberculosis (TB) caused by Mycobacterium tuberculosis infection is still threatening to human beings. It was reported that $\sim 9$ million people were infected and 1.4 million died from TB in 2011..$^{1}$ Several antibiotics, such as isoniazid, rifampicin, pyrazinamide, streptomycin, ethambutol, kanamycin and ethionamide, were developed as anti-TB drugs and three to four of them are usually used in combination for chemotherapy. ${ }^{2}$ However, new antibiotics with high activity are still desirable because of the emergence of multidrug-resistant TB. ${ }^{1}$

Pheganomycin (1) and resorcinomycin (2) were previously isolated as specific anti-Mycobacterium drugs from culture broths of actinomycetes. $^{3-5}$ Interestingly, neither compound is active against Gram negative bacteria and other Gram positive bacteria. Compound 1 consists of (S)-2-(3,5-dihydroxy-4-hyroxymethylphenyl)2-guanidinoacetic acid (3) at the $\mathrm{N}$-terminus and a proteinogenic core peptide derived from NVKDGPT or NVKDR. On the other hand, 2 is composed of a 3-related compound, (S)-2-(3,5-dihydroxy-4isopropylphenyl)-2-guanidinoacetic acid (4) and glycine (Figure 1). We recently identified a peptide ligase (PGM1) that catalyzed 3-phosphorylation with ATP and successive nucleophilic attack of the peptides. The enzyme showed broad substrate specificity and enabled us to make various 1-derivatives. ${ }^{6}$ In this study, we investigated the structure and activity relationships of $\mathbf{1}$ and $\mathbf{2}$ with these derivatives.

In our previous study, we enzymatically synthesized several 1-derivatives, all of which had (S)-2-guanidino-2-phenylacetic acid (5) as the $N$-terminal substrate. ${ }^{6}$ We first examined their antiMycobacterium activity with Mycobacterium smegmatis mc2 155 as a test strain by the conventional paper disk-agar diffusion assay. ${ }^{7}$ In all cases, most of the $\mathrm{N}$-terminal substrates were converted into products, and therefore the reaction products were directly used for the assay without purification. The concentrations of the products were calculated by quantitative HPLC analysis. ${ }^{6}$ We were unable to obtain enough amounts of the products (7 and $\mathbf{6}$ ) to weight the compounds. As an alternative approach, we measured and compared UV absorption at $271 \mathrm{~nm}$ of compounds 4 and $\mathbf{6}$ as references because UV absorption at $271 \mathrm{~nm}$ of the peptide (NVKDGPT) was negligible.
Molten soft agar containing the strain was spread on Luria-Broth agar plates. Paper disks containing various concentrations of the compounds were put onto the lawn of cells. After overnight incubation, the size of the halo was measured and the MIC was determined. However, none of the compounds tested showed activity regardless of the peptide-chain length or amino acid sequence of the peptides used as nucleophiles. These results suggested that the 3,5-dihyroxy groups and/or the moieties 4-hyroxymethyl in 1/4-isopropyl in $\mathbf{2}$ are essential for anti-Mycobacterium activity.

To investigate this possibility, (S)-2-(3,5-dihydroxyphenyl)2-guanidinoacetic acid (6) was chemically synthesized from 2-amino-2-(3,5-dihydroxyphenyl)acetic acid and formamidinesulfinic acid. ${ }^{8}$ Its structure was confirmed by NMR analysis (Supplementary Figure S1). We also tried purifying compounds $\mathbf{3}$ and $\mathbf{4}$ from the culture broth of a $p g m 1$-disruptant of the 1 producer ${ }^{6}$ and a $p g m 1$ ortholog (res5)-disruptant of the 2 producer, ${ }^{9}$ respectively. However, the amount of $\mathbf{3}$ accumulated in the culture broth of the disruptant was too small to purify and only 4 was purified. The thus obtained 4 and 6 were used for anti-Mycobacterium assays. However, neither compound exhibited activity (Table 1), showing that the attachment of at least one amino acid to the $N$-terminal substrate in the same way as 2 is required for anti-Mycobacterium activity.

As we were not able to detect enzyme activity of recombinant RES5, which is an ortholog of PGM1 and might catalyze the amide formation between $\mathbf{4}$ and glycine, ${ }^{9}$ we could not prepare a 2 -derivative composed of $\mathbf{6}$ and glycine. We therefore synthesized two compounds consisting of $\mathbf{4}$ or $\mathbf{6}$ at the $\mathrm{N}$-terminus followed by a heptapeptide (NVKDGPT) using recombinant PGM1. The reaction conditions were the same as those described previously. ${ }^{6}$ The formation of the respective compounds (7 and 8) was traced by LC-MS analysis (Supplementary Figure S2). The analytical conditions were described previously. ${ }^{6}$ Reaction product 7 , composed of 4 and NVKDGPT, showed almost the same activity as $\mathbf{1}$ (Table 1). In contrast, 8, consisting of $\mathbf{6}$ and NVKDGPT, did not show anti-Mycobacterium activity. These results suggested that the moieties 4-hyroxymethyl in 3 and 4 -isopropyl in $\mathbf{4}$ are essential for anti-Mycobacterium activity. 


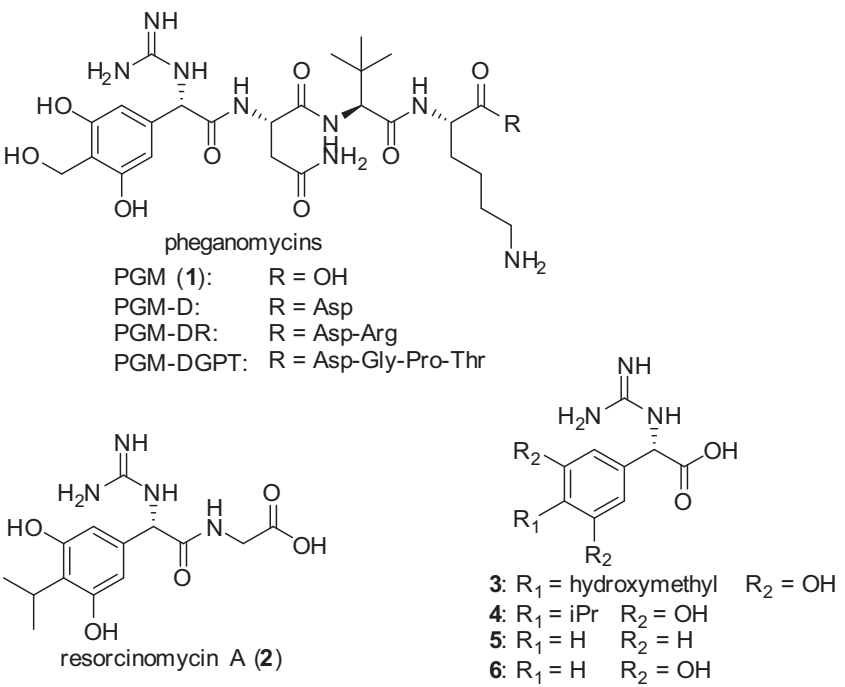

Figure 1 Structures of pheganomycins (1), resorcinomycin (2) and their corresponding $\mathrm{N}$-terminal nonproteinogenic amino acid derivatives in this study.

\section{Table 1 Bioactivity assay results against $M$. smegmatis}

\begin{tabular}{lc}
\hline Compounds & $M / C\left(\mu g /^{-1}\right)$ \\
\hline PGM (1) & 120 \\
Resorcinomycin A (2) & 20 \\
$\mathbf{3}$ & $>500$ \\
$\mathbf{4}$ & $>500$ \\
6 & $>500$ \\
NVKDGPT & $>500$ \\
7 & 200 \\
$\mathbf{8}$ & $>500$ \\
Spermidine & $>500$ \\
$\mathbf{9}$ & 200 \\
\hline
\end{tabular}

We next examined whether the peptide moieties of 1 could be replaced with other compounds. As PGM1 was suggested to have a long cleft to trap substrates by crystal structure studies, ${ }^{6}$ the enzyme perhaps recognizes a linear structure with an amino group at the $N$-terminus as a nucleophile. By screening candidate compounds with 4 as the counterpart substrate, we found that spermidine and spermine could be utilized as nucleophiles though the yields were low (Supplementary Figure S3). The product yield with spermine was especially low; therefore, the anti-Mycobacterium activity of compound 9, composed of 4 and spermidine, was examined. The reaction mixtures were concentrated by freeze-drying and re-dissolved in de-ionized water. After the product concentration was estimated by quantitative HPLC analysis, the sample was directly subjected to anti-Mycobacterium assay because spermidine and $\mathbf{4}$ showed no antiMycobacterium activities. As shown in Table 1, the product showed weak activity, suggesting that peptide structures in $\mathbf{1}$ are not essential for activity.

In this study, we investigated the structure and activity relationships of two anti-Mycobacterium antibiotics, 1 and 2. Using the broad substrate specificity of PGM1, which intrinsically catalyzed the amide bond formation between 3 and the heptapeptide (NVKDGPT), we synthesized unnatural $\mathbf{1}$ and $\mathbf{2}$-derivatives and examined their anti-Mycobacterium activities. Though the number of the tested compounds was small and their activities were rather weak, the following results were obtained in this study: (i) the attachment of at least one amino acid to the $N$-terminal substrate is essential because 4 itself has no activity; (ii) the moieties 4-hyroxymethyl in $\mathbf{3}$ and 4 -isopropyl in $\mathbf{4}$ are essential; (iii) the peptide moieties of $\mathbf{1}$ could be replaced with spermidine. Though the mechanism of action of both $\mathbf{1}$ and 2 remains uncertain, the results obtained in this study should be helpful for the development of $\mathbf{1}$ and $\mathbf{2}$-derivatives with high activity.

\section{CONFLICT OF INTEREST}

The authors declare no conflict of interest.

\section{ACKNOWLEDGEMENTS}

This study was supported by Grants-in-Aid for Scientific Research (25560397 and $15 \mathrm{H} 03110$ to T.D.) from the Japan Society for the Promotion of Science (JSPS). We thank Shionogi \& Co. Ltd (Osaka, Japan) and the Institute of Microbial Chemistry (Tokyo, Japan) for providing us resorcinomycin A and pheganomycin standards.

1 Paulson, T. Epidemiology: a mortal foe. Nature 502, S2-S3 (2013).

2 International Standards for Tuberculosis Care (ISTC) and the patients' charter for tuberculosis care, in WEB site of World Health Organization (2012). http://www.who.int/ tb/publications/2006/istc/en/ (accessed on 15 June 2015).

3 Suzukake-Tsuchiya, K., Hori, M., Shimada, N. \& Hamada, M. Mode of action of deoxypheganomycin D on Mycobacterium smegmatis ATCC 607. J. Antibiot. 41, 675-683 (1988).

4 Kondo, E. et al. Isolation and characterization of new antibiotics resorcinomycins $A$ and B. J. Antibiot. 42, 1-6 (1989).

5 Masaki, S., Konishi, T., Tsuji, N. \& Shoji, J. New antibiotics, resorcinomycins A and B: antibacterial activity of resorcinomycin A against mycobacteria in vitro. J. Antibiot. 42, 463-466 (1989).

6 Noike, M. et al. A peptide ligase and the ribosome cooperate to synthesize the peptide pheganomycin. Nat. Chem. Biol. 11, 71-76 (2015).

7 Raahave, D. Paper disk-agar diffusion assay of penicillin in the presence of streptomycin. Antimicrob. Agents Chemother. 6, 603-605 (1974).

8 Jursic, B. S., Neumann, D. \& McPherson, A. Preparation of $\mathrm{N}$-formamidinylamino acids from amino and formamidinesulfinic acids. Synthesis 32, 1656-1658 (2000).

9 Ooya, K., Ogasawara, Y., Noike, M. \& Dairi, T. Identification and analysis of the resorcinomycin biosynthetic gene cluster. Biosci. Biotechol. Biochem. (2015). doi:10.1080/09168451.2015.1050992.

Supplementary Information accompanies the paper on The Journal of Antibiotics website (http://www.nature.com/ja) 